\title{
ANTECEDENTES EDUCATIVOS DE LA MUJER EN MÉXICO Y EN EL ESTADO DE TABASCO
}

\author{
Josefina De la Cruz Izquierdo ${ }^{1}$ \\ Laura López Díaz ${ }^{2}$ \\ José Antonio Morales Notario ${ }^{3}$
}

Resumen: La mujer ha ido caminando a tropiezos y golpes bajos para ir logrando abrirse camino en espacios que se le han cerrado por cuestiones de género. El aspecto social y cultural han influido enormemente para que en materia educativa le haya costado trabajo el prepararse y desarrollarse personalmente. A pesar de todos esos contratiempos y sinsabores ha logrado un espacio que orgullosamente proclama para colocarse en situaciones de liderazgo que instan a las demás mujeres en esa búsqueda de empoderamiento que les brinde un mejor desarrollo y aporte una equidad en la sociedad. De ahí la importancia de resguardar y difundir este doloroso proceso de lucha y sobrevivencia, pero pleno de perseverancia y motivación, por lo que el objetivo de esta investigación radica en hacer un recuento del proceso educativo en donde se incluyó a la mujer y su desenvolvimiento en ello y lo que eso influyó en su desarrollo personal de la mujer en México, lo cual se realizará por medio de esta investigación de tipo cualitativa y bibliográfica.

Palabras Claves: Educación, igualdad, universidad.

\begin{abstract}
Women have been moving their path through stumbles and low blows in order to achieve a place in society that has been denied by gender matters. The social and cultural aspects have had enormous influence in the educational topic because
\end{abstract}

\footnotetext{
${ }^{1}$ Profesora Investigador en la División Académica de Ciencias Sociales y Humanidades de la Universidad Juárez Autónoma de Tabasco en México. Líder del grupo de investigación "Derechos Humanos y Responsabilidad Social".

${ }^{2}$ Profesor Investigador de Tiempo Completo Asociado "A" en la División Académica de Informática y Sistemas de la Universidad Juárez Autónoma de Tabasco en México. Integrante del grupo de investigación "Derechos Humanos y Responsabilidad Social"

${ }^{3}$ Profesor Investigador de Tiempo Completo Asociado "A" en la División Académica de Ciencias Sociales y Humanidades de la Universidad Juárez Autónoma de Tabasco en México. Integrante del grupo de investigación "Derechos Humanos y Responsabilidad Social"
} 


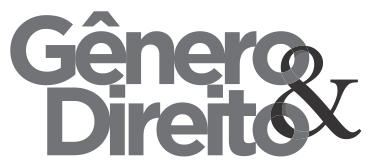

society had presented obstacles and education had been denied to allow women to improve themselves. Despite of all these setbacks, they have proudly gained a space to put themselves into leading positions that empower others and motivate them to seek for their own development and improvement and provide an equal aspect to society. Therefore, the importance of cocoon their history and spread them to let people know all the struggles and sorrows they have survived, but also all the perseverance and motivation stories; that is the reason why this paper makes an analysis of the educational process view through women's eyes and their development that influenced in their personal progress which is made though this research of qualitative and bibliographic style.

Palavras Chaves: Educação, igualdade, universidade

\section{INTRODUCCION}

La UNESCO (2016) considera que la educación es un derecho humano para todos, a lo largo de toda la vida, y que
Cédico do Núcleo de Estudos e Pesquisas sobre Gênero e Direito Centro de Ciências Jurídicas - Universidade Federal da Paraíba V. 6 - No 02 - Ano 2017

el acceso a la instrucción de ir acompañado de la calidad.

El determinar la importancia de que las mujeres realmente puedan acceder a los estudios superiores a pesar de los miles de obstáculos a los que se enfrenta en su diario vivir brinda una perspectiva del avance que como sociedad democrática manejamos.

El propósito de esta investigación fue realizar un análisis de la visión sobre el ámbito de las mujeres que han luchado por la educación y las necesidades que manejan en su vida diaria, ya que deben lidiar con situaciones del ámbito hogareño como puede ser el control y manejo de la economía doméstica, así como la atención y cuidado del esposo y los hijos, y todavía anexar sus obligaciones laborales, pues la economía nacional no permite que un solo ingreso cubra ciertas necesidades básicas en el hogar, motivo por el cual ya las chicas desde jóvenes y solteras trabajan, primero para apoyar a sus padres y después para apoyar a su propia familia.

Esta investigación dará por lo tanto una perspectiva impersonal sobre las barreras e inconvenientes que han venido viviendo las mujeres durante años y que actualmente siguen sufriendo por causa de 


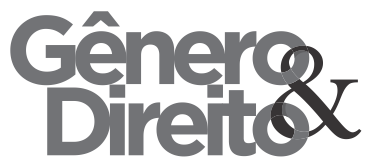

género y acciones que realizan para lograr un correcto desenvolvimiento académico y contar con el apropiado apoyo institucional y familiar o los métodos para lidiar con toda esa problemática para lograr solventar su desarrollo.

Desafortunadamente por el rol tradicionalista de la división del trabajo, a las mujeres se les ha asignado la responsabilidad del cuidado, protección de los hijos y la realización de actividades del hogar, lo cual las limita a un pequeño círculo social que no permite totalmente la participación de las mujeres en actividades al cien por ciento como lo es la educación.

Es por ello que a continuación se mencionan antecedentes históricos y vivencias de mujeres con la finalidad de mostrar un panorama real de la concepción de las mujeres que ha luchado en la educación.

\section{LOS DERECHOS DE LA MUJER.}

Las diferencias existentes en el trato hacia la mujer siguen preponderando hoy día; la mujer sigue sufriendo trato desigual y sistemáticamente siguen violentado sus derechos, a pesar de los diversos instrumentos internacionales que dico do Núcleo de Estudos e Pesquisas sobre Gênero e Direito Centro de Ciências Jurídicas - Universidade Federal da Paraíba

V. 6 - $\mathrm{N}^{\circ} 02$ - Ano 2017

158

han sido creados por las naciones interesadas en resarcir un poco del daño realizado por medio de reglas claras y precisas, como en el caso de la "Convención de Belem Do Pará"; instrumento internacional adoptado por México el 9 de junio de 1994, y ratificado por el Senado el 12 de noviembre de 1998, para entrar en vigor el 12 de diciembre de ese mismo año (Convención Interamericana de Derechos Humanos, (1994).

En este Convenio se especifica que los países han reconocido a las mujeres como víctimas de la violencia, por lo que sus libertades fundamentales se encuentran limitadas de manera parcial o total, lo que en realidad preocupa en demasía a la Comunidad Internacional, pues esta situación ofende a la dignidad humana y prueban la existente relación de poder de los hombres sobre las mujeres. Pero sobre todo insta a que se dé una búsqueda de modificación de patrones socioculturales no sólo en el hombre sino también entre las mujeres como un medio de eliminación de prácticas educativas que solo refuerzan y promueven las ideas y actitudes estereotipadas de violencia contra las mujeres. 


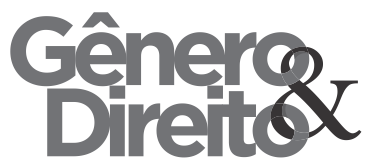

Dentro de esa investigación se enfatiza el fomento a la educación y capacitación de agentes de Estado para poder aplicar las leyes y políticas de prevención, sanción y eliminación de violencia contra las mujeres de manera apropiada y la educación para la población en general que permita que las mujeres y niñas tengan acceso a los programas educativos y a la búsqueda de mejora personal y acceso a un mejor estilo de vida (CIDH, 1994).

\section{ANTECEDENTES DE LA MUJER EN} LA EDUCACIÓN

La enseñanza de siglos atrás fue influenciada grandemente por la iglesia, la cual consideraba que la mujer no podía manejar puestos de liderazgo y que solo debía estar rezagada a papeles secundarios en donde solo podían realizar ciertas tareas como la del cuidado de los niños y el mantenimiento del hogar, proveyendo de atención y cuidado a cada miembro de la familia sin prestar atención a sus propias necesidades. Sin embargo, algunas de ellas lograron desafiar el control religioso y alcanzaron avances significativos en diversos campos, como la española Oliva
Céco do Núcleo de Estudos e Pesquisas sobre Gênero e Direito Centro de Ciências Jurídicas - Universidade Federal da Paraíba V. 6 - $\mathrm{N}^{\circ} 02$ - Ano 2017 159

Sabuco de Nantes (1562-1588) quien fue filósofa y médica; Mary Wortley Montagu, quien descubrió la vacuna contra la viruela; Emilie du Chatelet, matemática y física francesa que vivió de 1706 a 1749 y que propagó los descubrimientos de Newton; María Andrea Casamayor y de la Coma, española investigadora sobre aritmética cuyos estudios ayudaron a los investigadores de las matemáticas aplicadas; entre otras.

En México, Sor Juana Inés de la Cruz, fue la mujer más reconocida, pero tuvo que ingresar a un convento debido a su negativa de contraer matrimonio y a pesar de eso, vio en esa opción la posibilidad de continuar sus estudios y seguir preparándose.

Juana de Asbaje, como también se le conocía fue mujer de mente prolífica que ha sido siempre el ejemplo más utilizado cuando se refiere a la educación en los años 1600. Al ingresar al convento debía dedicarse plenamente a la vida consular, pero encontró en ella la satisfacción de poder tener acceso a la educación que tanto anhelaba y logró prepararse en los ámbitos literarios principalmente. Su obra más reconocida es su "Carta Atenagórica de la madre Juana 


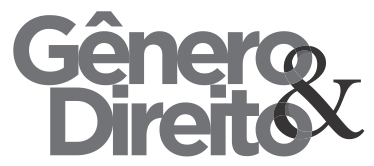

Inés de la Cruz, Religiosa profesa de velo y coro en el muy religioso convento de San Jerónimo", en la que le responde a Sor Filotea de la Cruz, que era el pseudónimo utilizado por Manuel Fernández De la Cruz, un Obispo de Puebla, quien previamente había criticado un sermón del jesuita portugués Antonio de Vieyra, teólogo, sobre las finezas de Cristo.

Y debido a eso, en "Carta de Sor

Filotea de la Cruz" se admitió el derecho que las mujeres tienen de acceder a la educación, pero en específico se le recomendó que se dedicara a la vida monástica, puesto que en su condición de monja y mujer sólo esos eran sus derechos, pero, se le especificaba que la reflexión teológica estaba reservada sólo para los hombres (México Desconocido, 24 de febrero de 2014).

\section{Continuando con los} antecedentes del acceso a la educación por parte de las mujeres, en los años 1800's, el presidente Comonfort ordenó la fundación del primer colegio de educación secundaria para niñas, en contra de los liberales de la época que se oponían a este beneficio, ya que, influiría en la mentalidad de las mujeres volviéndolas más "rebeldes e insubordinadas". Sin
Cico do Núcleo de Estudos e Pesquisas sobre Gênero e Direito Centro de Ciências Jurídicas - Universidade Federal da Paraíba V. 6 - $\mathrm{N}^{\circ} 02$ - Ano 2017 160 embargo, se discriminó este acceso por cuestiones económicas, pues sólo se proporcionó a aquellas jóvenes de clase acomodada. Pero en 1875 se introdujo la educación pedagógica en la Escuela Nacional Secundaria de Señoritas, convirtiéndose en Escuela Normal.

Cualquiera pensaría que con tantos avances e instrumentos internacionales las mujeres tienen acceso a sus derechos básicos y fundamentales, pero por a como lo menciona Morales, $\mathrm{T}$. (2015) existe el caso de Malala Yousafzai (nacida apenas en 1997), quien al igual que miles de mujeres, libra en la actualidad la misma batalla que vivió Sor Juana por el derecho a la educación. Nacida en Pakistán, se vio obligada por el régimen talibán a dejar la escuela. Los talibanes forzaron el cierre de las escuelas privadas y prohibieron la educación de las niñas entre 2003 y 2009. Ella se identifica como una firme activista y defensora de los derechos de las mujeres, sobre todo en el aspecto educativo, razón por la cual, Malala recibió varios disparos, de parte del grupo talibán, mientras se encontraba en el autobús escolar.

Además de denunciar al grupo religioso que la atacó y defender sus 


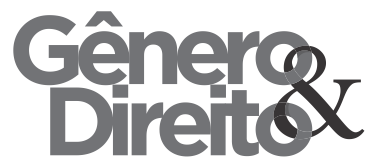

derechos educativos y los de otras niñas pakistaníes, Malala es hoy una activista, de importancia mundial. En mayo de 2014, participó en una campaña para la liberación de un grupo de jóvenes nigerianas secuestradas mientras estudiaban, por un grupo islamita que continúa rechazando el derecho de las mujeres a la educación.

El 10 de diciembre de 2014, la joven pakistaní recibió, al igual que Kailash Satyarthi, el Premio Nobel de la Paz, convirtiéndose históricamente, en la ganadora con menor edad de este premio. A partir de su nominación, Malala ha sido entrevistada por medios de todo el mundo, ha publicado un libro y ha pronunciado varios discursos en los que reitera su compromiso con las mujeres y sus derechos.

\section{LA INCLUSIÓN DE LA MUJER EN LA EDUCACIÓN SUPERIOR EN MÉXICO.}

La época de la Revolución Mexicana tuvo una influencia en la mujer mexicana, ya que empezó a visualizarse como parte de la esfera pública y tomar un papel más allá del correspondiente a las
Cédico do Núcleo de Estudos e Pesquisas sobre Gênero e Direito Centro de Ciências Jurídicas - Universidade Federal da Paraíba V. 6 - No 02 - Ano 2017

labores del hogar y cuidado de los hijos del matrimonio.

El hecho de volverse soldaderas le facilitó los espacios para movilizarse en cualquier ámbito, pues debían alimentar a sus "hombres" por lo que accesaban a todos los lugares por cualquier medio, ya sea por amistad, compra-venta o favores sexuales. El hecho de seguir a los hombres, les permitía aprender todo lo relacionado al cuartel, llegando incluso a portar armas y pelear lado a lado de los hombres en el ejército revolucionario. Esta experiencia migratoria, cargada de malos tratos y vejaciones, las forzó a adaptarse a las situaciones que les tocaban e ingresar en nuevos puestos y ocupaciones, logrando ser despachadoras de trenes, telegrafistas, enfermeras, farmacéuticas, empleadas de oficina, reporteras, editoras de periódicos, mujeres de negocios y maestras. En más de una ocasión demostraron sus aptitudes y actitudes en defensa de los hombres con los que luchaban, lo que la hizo ganar reconocimiento como compañera, consorte y pareja. También en esta época se recuerdan los innumerables hechos que buscaban el reconocerle su derecho al voto y su participación en la vida política (Lavalle Urbina, M. (1988)). 


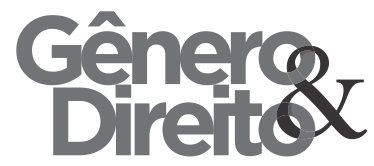

Como se mencionó

anteriormente, ya para el año 1823 se dan los primeros antecedentes de la educación laica en México. En 1868 con la promulgación de la Ley de Instrucción Pública se apertura la enseñanza para niñas en donde se incluían asignaturas normales como moral y urbanidad. Pero el cursar el bachillerato todavía presentaba problemas, lo mismo que para la superior. En los años de 1870 y 1890 egresaron las primeras mujeres con secundaria y las primeras profesoras, siendo las normales el uno de los dos espacios de desarrollo profesional e intelectual permitido para las féminas que no tenían en sus planes el matrimonio o el tener hijos, pues era considera una continuidad del rol que se le atribuía. El otro espacio era el del ámbito de la enfermería pues se le consideraba como madre-protectora o cuidadora, lo que se instalaba dentro del estereotipo establecido para la mujer.

Para fines del siglo XIX existieron escuelas de Artes y Oficios para Mujeres, pero se enfocaban a bordado, costura, teneduría de libros, pasamanería, tapicería, flores artificiales, dibujo y pintura, música, encuadernación y tipografía, pero con algunas clases
Céco do Núcleo de Estudos e Pesquisas sobre Gênero e Direito Centro de Ciências Jurídicas - Universidade Federal da Paraíba V. 6 - $\mathrm{N}^{\circ} 02$ - Ano 2017 162

referentes a la virtud cristiana femenina y temas religiosos de comportamiento adecuado (Rodríguez Mancera, M. (s. f.)).

Aunque no fue fácil la incursión de la mujer dentro de los ámbitos de estudios superiores con la Escuela Nacional Preparatoria y estar manipuladas las asignaturas para que continuasen siendo una extensión del rol "correspondiente" a la mujer, para 1910 con la inauguración de la Universidad Nacional Autónoma de México y a pesar que existía una acuerdo para permitir el ingreso a las mujeres, muy pocas se atrevieron, por lo que en esa época se graduaron muchas maestras, algunas abogadas, médicas y dentistas. Esto se reflejó en la mala aceptación dentro de la institución pues eran mal vistas y criticadas, y aún a pesar de eso, en 1887 se recibió la primera mujer médica y en 1898 se graduó la primera abogada y para 1909 la primera dentista.

En esa época y según los datos de Anuario Estadístico mencionado por Córdova Osnaya, M., (1940-1958) las mujeres eran el $20.73 \%$ en 1940 , el $18.26 \%$ en 1950 y el $17.62 \%$ en 1960 .

Para los años 80's, y de acuerdo a la ANUIES, el $30 \%$ de la población 


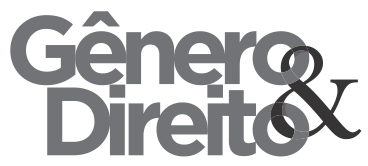

estudiantil en la universidad eran mujeres

y esta cifra aumentó para 1984 llegando a un $32 \%$, dedicándose al estudio de las Ciencias Sociales, Administrativas y de la Salud y muy pocas para Ciencias Agropecuarias, Ingeniería y Tecnología, así como la mitad de la matrícula para las de Educación y Humanidades. Estas cifras no cambiaron mucho para los años 90's, sin embargo, para el año 2000 es que las cifras de las mujeres empiezan a incrementar e incluso a superar a la población masculina (Córdova Osnaya, M., (s. f.)).

No fue sino hasta 1884 , en que Luz Bonequi, una de las precursoras en desarrollarse en otras actividades laborales completamente diferentes a las establecidas por la sociedad y la religión, obtuvo el primer título de telegrafista para una mujer y logró colocarse en la Oficina Central de Telégrafos. Posterior a ella, se destacó Margarita Charné, (1866), al titularse como la primera mujer dentista. Para el 1887, Matilde Montoya se vuelve la primera médico cirujano y para 1889 , María Asunción Sandoval de Zarco obtuvo su título de abogada (Trillo Tinoco, F., (2004).
Céco do Núcleo de Estudos e Pesquisas sobre Gênero e Direito Centro de Ciências Jurídicas - Universidade Federal da Paraíba V. 6 - $\mathrm{N}^{\circ} 02$ - Ano 2017 163

Es triste saber que en muchas comunidades de los estados de México se sigan dando estas costumbres de limitación hacia las mujeres, ya que las rezagan por cuestiones de género en el que, acorde a sus ideas, está implícito el que no ejercerán los estudios realizados y que no vale la pena el esfuerzo en prepararlas y educarlas si su lugar corresponde al hogar y al cuidado de los hijos. Incluso consideran que el hecho de enviarlas a realizar sus estudios universitarios lo único que lograran será el envolverse en un ambiente "desenfrenado y libertino" que sólo la dejará con un sabor amargo, que la harán caer en un compromiso matrimonial sin prerrogativas ni derechos o quedar con el compromiso de un hijo y sola con el compromiso maternal, por lo que bajo estas premisas manipulan y convencen a sus hijas de ceder ese anhelo de superación personal.

\section{LA INCLUSIÓN DE LA MUJER EN LA EDUCACIÓN SUPERIOR EN TABASCO.}

Como se mencionó en los anteriores estudios, posterior a la época de la Revolución Mexicana, pero con 


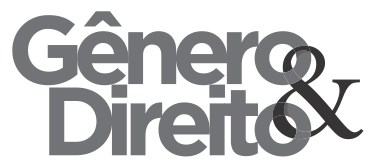

referente al estado de Tabasco y durante el periodo del presidente Venustiano Carranza, se nombró a Francisco J. Múgica como gobernador del Estado, el cual creó un programa que se denominó “La República Escolar”, que implicaba la supresión de toda enseñanza religiosa en las escuelas y se aprovecharan las iglesias como escuelas. Es ahí donde se funda la escuela vocacional dirigida a la mujer, fue en las mismas fechas que la capital cambio de San Juan Bautista a Villahermosa. Cuando termina su mandato el gobernador Múgica, se disputan el poder dos grupos políticos: los Rojos con Carlos Greene a la cabeza y el partido Radical Tabasqueño apoyándolos y los Azules encabezado por Luis Felipe Domínguez y el Partido Liberal Constitucionalista, de donde surgió como ganador el grupo de los Rojos, colocando como gobernante interino a Tomás Garrido Canabal.

Este personaje tan reconocido en Tabasco estuvo por 15 años en el poder, apoyado por Obregón y después apoyó a Plutarco Elías Calles, dividiéndose Garrido contra Carlos Green, los cuales al ser derrotados tienen que irse a la Ciudad de México y en la época del Maximato, Garrido tiene gran libertad, sin tener dico do Núcleo de Estudos e Pesquisas sobre Gênero e Direito Centro de Ciências Jurídicas - Universidade Federal da Paraíba V. 6 - No 02 - Ano 2017

intervención del centro por lo que logró un desarrollo económico muy importante.

Es durante esta época y con las ideas del gobernador basadas en un radicalismo ideológico que se basaba en la escuela racionalista del anarquista español Francisco Ferrer Guardia, la cual fue difundida en México por José de la Luz Mena, la cual tenía por objetivo el desarrollo del niño sin prejuicios ideológicos a fin de crear personas verídicas, justas y libres, tratando de arrancar de raíz los prejuicios de clase, por lo que esa temporada se conoció como una época de anti-religionismo y de gran lucha anticlerical.

Es ahí donde se inicia a incluir a la mujer, pero solo en el ámbito escolar, pues podía desarrollarse como institutriz o profesora, recibiéndose mucho apoyo por parte del magisterio veracruzano con referencia a capacitación y mejora profesional (Badía-Muñoz, Graciela Isabel, Pedrero-Nieto, Gloria (2015)).

Este ámbito profesional fue apoyado por el Instituto Juárez que se instauró por iniciativa de Benito Juárez, que destinó de los fondos para el clero un porcentaje que permitiera el desarrollo del proyecto educativo. Al inicio se le 


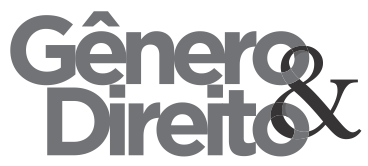

denominó Colegio Juárez y se le destinaban seis mil pesos mensuales y se le formaría un fondo con los capitales y réditos de la nacionalización de los bienes eclesiásticos.

Para el $1^{\circ}$ de enero de 1879 con el Dr. Simón Sarlat Nova se emite un decreto el 12 de noviembre en 1878 para dar a conocer el reglamento y Plan de estudios del ya Instituto Juárez, el cual establecía que se iniciaría con dos secundarias o preparatorias y superior o profesional. A la preparatoria se le dedicaban cinco años de estudios con el propósito de capacitar ciudadanos integrales y con perspectiva científica que los preparara para sus estudios profesionales. Como opciones profesionales se ofrecían las carreras de Pedagogía, Notariado, Comercio, Agrimensura, Agricultura y Veterinaria, actividades a las que se encaminaba la economía del estado y a todas vistas enfocadas hacia la población masculina. El instituto aceptaba 15 estudiantes en calidad de becario, costeados por el Estado y la manutención se manejaba en $\$ 250.00$ anuales. Cada municipio tenía derecho a enviar un alumno de condiciones muy pobres escogido de entre los mejores y de mejor desempeño escolar previo. Centro de Ciências Jurídicas - Universidade Federal da Paraíba V. 6 - $\mathrm{N}^{\circ} 02$ - Ano 2017

165

Para el período de 1904 se formó

la Escuela Normal para Profesoras, siguiendo el precepto de continuar con su labor de forjadora y reproductora ideológica. Sin embargo, su educación estuvo limitada, para su instalación se gastaron solamente $\$ 5,360.00$, se le permitió solo 17 alumnos a las cuales se les otorgaban \$16.00 para su manutención por un período de 10 meses.

La instrucción se dividía en primaria elemental y la instrucción primaria superior. La primera se realizaba en un periodo de tres años, tiempo en el que se le incluían asignaturas como labores manuales, corte y confección, dibujo, música y canto entre algunas de conocimiento general. Para tener acceso al plan de estudios de instrucción superior debían llevar dos años complementarios a su instrucción. Se les cuidaba desde los libros que se les facilitaba para su aprendizaje, hasta su salud (Torres Vera, M., y Romero Rodríguez, L., (s. f.).

Para 1936, con el gobernador Víctor Fernández Manero se reorganiza el Instituto Juárez y se separa la secundaria de la preparatoria, iniciando los 40's con bachilleratos en Ciencias Sociales y Biología. Para el periodo de Noé de la Flor 
Casanova aprueba reformas para consolidad los planes de estudio del bachillerato, restituir la Escuela Normal y mejorar la biblioteca universitaria José Martí, contando con una matrícula de 200 alumnos, pero lucharon contra la inestabilidad económica imperante en el estado. Cuando toma el control Francisco J. Santamaría del estado y en el Instituto Juárez se instala como director Belisario Colorado Jr., se inicia una reforma que moderna y fortalece la preparatoria, se funden cuatro escuelas secundarias y se reabre la carrera de Jurisprudencia, al igual que crea un sistema de becas para la Escuela Normal y se incorporan a la Asociación Nacional de Universidades e Instituciones de Enseñanza Superior. Para ese entonces se impartían las carreras de Comercio, Jurisprudencia, Medicina Veterinaria y Enfermería, por lo que para el 20 de noviembre de 1958 se concreta como universidad.

En ese tenor $y$ por tanta renovación el instituto empezó a ser más estricto con las especificaciones de los profesores que contrataba para impartir clases y en 1952 empezó a exigir que presentaran su título profesional y demostrar tres años de ejercicio y experiencia o ser profesor de educación primaria y no pertenecer como ministro de algún culto religioso, y es en este apartado donde se consideraba incluso la opción de admitir a las mujeres para impartir cátedras, siendo requisito que no estuviesen en estado de gravidez o en caso contrario, solo se le permitiría laborar hasta el cuarto mes de embarazo y después de haber concluido la lactancia (Pérez Castro, J., ( 2015)).

La apertura de inclusión de las mujeres en el ámbito laboral y educativo fue restringida a los ámbitos que consideraban como los referentes a su género: educar y cuidar; por lo tanto, por mucho tiempo el magisterio fue desempeñado principalmente por mujeres y en todos los niveles jerárquicos posibles. Es en este espacio en el que cabe mencionar un ícono educativo en la región y que es muy reconocido: Rosario María Gutiérrez Eskildsen, profesora, poeta y profesora de lingüística, que logró impactar a personajes de gran realce en el Estado con su compromiso, su empeño y desenvolvimiento, logrado a través de sus estudios pues fue titulada de Maestra en Ciencias de la Educación y un Doctorado en Letras de en la subsección de 
Lingüística Románica (Pérez Morales, F., Castillo Guzmán, R., Gorrochotegui Salas, M., y Estrada Sánchez, C. (2008)).

Todo el proceso revolucionario vivido por las mujeres ha determinado que aún con el paso del tiempo, no ha habido mucho cambio y modificación en las situaciones de rezago que las mujeres viven, principalmente en aquellas que ya están casadas. Cuando se encuentran en esa situación, forma un hogar con la idea preconcebida que seguir estudiando significa descuidar sus obligaciones, y algunas otras mujeres, por situaciones fuera de su alcance, deben trabajar para apoyar en el sustento familiar; por esa razón, muchas mujeres han visto en el desarrollo de las tecnologías, una oportunidad por medio de la cual pueden desenvolverse y educarse.

\section{IGUALDAD DE GÉNERO EN LA EDUCACIÓN VIRTUAL.}

La educación a distancia es el proceso educativo, con flexibilidad en cuanto a la asistencia a clases, el calendario escolar y las fechas de evaluación de lo aprendido, más no así, de la documentación de dichos avances
(Moreno, M., 2006). Todas las actividades de interacción con el contenido y la institución y el mismo asesor se manejan en diferentes espacios y tiempos.

Esta modalidad surgió debido a la imposibilidad de las instituciones de albergar a más estudiantes de manera física y el desarrollo de nuevas tecnologías permitieron les permitió llevar la educación a aquella población que por falta de tiempo y medios no podían cursarla. Sus primeros inicios fueron a través del correo y el radio para consultas individuales y grupales (cursos por correspondencia, cursos orales complementarios, centros locales de estudio y consulta, etc.).

En México, en 1979, la Universidad Pedagógica Nacional cubrió al país con este tipo de estudios, pero continuaban manejando asesorías sabatinas con profesores y estudiantes. En los años 90, cuando el internet se expandió, se permitió que la modalidad a distancia encontrase un espacio excelente en la educación superior que permitiese a los alumnos el acceso a la información que requerían en menos tiempo y en diferentes momentos y permitió a los asesores llevar un seguimiento de los alumnos y mantener 


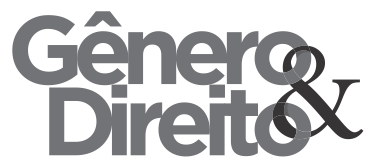

Gônerger

una comunicación constante (Moreno, M.; 2006).

A pesar de la facilidad con que se puede hacer uso de la educación por medios virtuales, existen limitaciones que se deben contrarrestar. Uno de los más importantes se refiere a la lucha interior y discreta que libran las mujeres por mejorar su autoestima y considerarse capaces y eficientes para cambiar su situación actual y contrarrestar todas esas ideas preconcebidas que la cultura les ha impuesto por años donde las definen como alguien falto de capacidades y destrezas e incapaz de realizar un aprendizaje efectivo.

La independencia financiera para tener acceso a una computadora es otro reto, pues es difícil costear y tener una en casa. Si lo logra, no puede trasladarla de un lugar otro, porque se arriesga a perderla y con ella su información; por lo que los lugares de renta son una excelente opción, pero carentes de privacidad, ya que son de uso público. La renta del uso del internet es otro limitante, pues si no trabaja se encuentra supeditada a la decisión del cónyuge de pagar el servicio o no y se presta a chantaje o manipulación. Las mujeres se encuentran en desventaja y
Cen do Núcleo de Estudos e Pesquisas sobre Gênero e Direito Centro de Ciências Jurídicas - Universidade Federal da Paraíba

V. 6 - $\mathrm{N}^{\circ} 02$ - Ano 2017

168

muchas veces limitadas por el analfabetismo tecnológico, por no permitírsele su uso, práctica y manejo. El uso limitado de la computadora no le permite practicar adecuadamente y recabar la información que necesita para su estudio, así como el conocer diversos tipos de software que se utilizan en la red. Este analfabetismo provoca que su desempeño se vea empañado y se vuelva en algunas ocasiones negativo, afectando a su autoestima y disminuyendo su motivación lo cual concluye con el abandono de sus metas y objetivos.

Otro inconveniente al que se enfrentan es el denominado "trabajo fantasma" (término utilizado por Ivan Illich) o la inequidad en la distribución de actividades de la casa, puesto que por cuestiones culturales, ella está a cargo de la carga de la atención de los hijos y los integrantes de la familia, la elaboración de alimentos y cuidados del hogar, el cuidado de los enfermos y adultos mayores, al igual que la disponibilidad de la ropa para los integrantes de la familia, por lo que la oportunidad de trabajar en su desempeño académico se relega a sus verdaderos tiempos de descanso y reposo. $\mathrm{Y}$, aún a pesar de todas estas situaciones, por su 


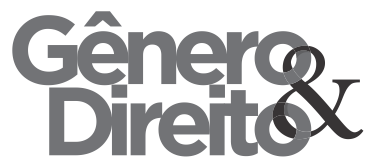

misma motivación intrínseca y deseo de superarse para mejorar su situación personal, las mujeres se esfuerzan más para tener un mejor desempeño, organizando su tiempo y actividades y manejando una mejor actitud (Gayol, Y., (2015).

ESTADÍSTICAS CON REFERENCIA A LA EDUCACIÓN

De acuerdo a los datos obtenidos del INEGI en el año 2000, por cada 100 mujeres, 12 no tuvieron acceso a la instrucción, 43 tenían al menos un grado aprobado en primaria y uno o dos años de secundaria; 18 aprobaron tres grados de secundaria y 27 tenían estudios pos básicos. Esta problemática es más fuerte cuando la situación se desenvuelve en comunidades rurales con menos de dos mil 500 habitantes, en donde de cada 100 hombres 16 no tenían instrucción, y de cada 100 mujeres 21 se encuentran en la misma situación.

A esto se anexan los datos recopilados en el Instituto Nacional de las Mujeres (INMUJERES) que señala una tasa de analfabetismo para la población femenina de un 11.3 por ciento, mientras
Codico do Núcleo de Estudos e Pesquisas sobre Gênero e Direito Centro de Ciências Jurídicas - Universidade Federal da Paraíba V. 6 - $\mathrm{N}^{\circ} 02$ - Ano 2017

que la masculina es de 7.4. Anexando a eso el que la población femenina que reside en zonas rurales tiene 4.6 años de estudio promedio, en contraste con las que viven en zonas urbanas que tienen 7.9 años.

Pero, según el Consejo Nacional de Población refiere que, en la actualidad en México, las y los jóvenes cuentan con niveles educativos mayores que las generaciones que las antecedieron. Además, en sólo tres décadas la proporción de personas de 15 a 24 años que finalizó la educación media superior o realizó estudios superiores, aumentó de poco menos de 10 por ciento a casi 30 por ciento. Así, la mayor permanencia en la escuela se relaciona con una menor participación económica de parte de los adolescentes, que se vio disminuida de 60.3 a 49.1 por ciento entre 1995 y 2003 en el caso de los hombres, y de 29.6 a 24.2 por ciento en el de las mujeres. A pesar de ésta disminución en los indicadores, los niveles educativos de México comparados con los países más desarrollados se presentan en gran desventaja.

Posterior a este panorama de desarrollo educativo, un estudio realizado en el año 2000 por la Asociación Nacional de Universidades e Instituciones de 


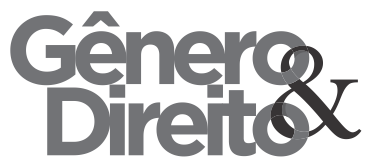

Educación Superior (ANUIES), reveló que en el Distrito Federal existían 394 mil estudiantes inscritos en licenciatura $\mathrm{y}$ postgrado, y que a pesar del gran número, solamente se podrá extender una plaza disponible para cada 3.76 mujeres y hombres que concluyan sus estudios.

En ese contexto, la ex directora general de la ANUIES, Diana Ortega, manifestó lo complacida que estaba, ya que la participación femenina en este nivel educativo ha tenido notables avances, y esto se ve reflejado en un cambio en las expectativas sociales y personales de las mismas.

Al mismo tiempo manifestó que en los años 70's en México, las mujeres que estudiaban la licenciatura estaban representadas por un 17 por ciento de la población escolar, mientras que en el año 2001 este número se incrementó hasta el 48 por ciento.

Aunque este incremento no necesariamente es un indicador de equidad, puesto que las proporciones son diversas acorde a las áreas de conocimiento en que se desenvuelven, siendo un ejemplo de esto el aspecto referente a la investigación científica de la UNAM, ya que de los dos mil 368
Codico do Núcleo de Estudos e Pesquisas sobre Gênero e Direito Centro de Ciências Jurídicas - Universidade Federal da Paraíba V. 6 - No 02 - Ano 2017 170 investigadores existentes en ese momento, sólo el 24 por ciento de éste son mujeres.

Este factor incidente en el desarrollo de la educación se debe a que en el año 2000, un 0.43 por ciento del Producto Interno Bruto (PIB) era asignado a la educación, y aunque el índice poblacional se incrementaba y se ha seguido incrementando, solamente se le ha aumentado a un $0.58 \%$ del PIB a este sector de la sociedad de acuerdo a la información presentada por el periódico "El Universal" (2002). Anexo a estos indicadores se compagina una declaración hecha por la UNESCO, donde denomina a México como uno de "los gigantes del analfabetismo mundial", comparándolo de igual forma que a China, India, Bangladesh, Pakistán, Indonesia, Nigeria, Egipto y Brasil. Todo esto se comprueba con los índices encontrados en un estudio realizado en el año 2000 que determina que mientras un hombre percibía un promedio de $\$ 40.94$ pesos por hora una mujer únicamente percibe $\$ 26.70$ en promedio, aunque "ambos sean profesionistas" y realzando así la discriminación que aún se sigue propagando dentro de nuestra sociedad 


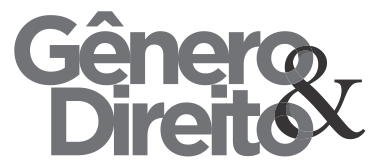

mexicana de acuerdo a los datos del INEGI (1999) (Nadeskko, (2010)).

Con referencia a los indicadores existentes es el estado de Tabasco, en lo que respecta a la Universidad Juárez Autónoma de Tabasco, se encontró según informe reciente del rector del mismo instituto Piña Gutiérrez, J. (2017), que se inscribieron un total de 16, 779 mujeres de un total de 30,262 en el nivel de Técnico Superior Universitario, Licenciaturas y Posgrados, específicamente en la modalidad escolarizada, siendo éste un 55 por ciento del total de alumnos; mientras que en la modalidad no escolarizada, de un total de 665 estudiantes inscritos, 460 fueron mujeres, siendo éste un 69 por ciento del total de alumnos. Por lo que en total de todo el matriculado en la universidad se representó una cifra de 32, 093 mujeres inscritas de un total de 56,400, siendo representado este porcentaje de un 57 por ciento de mujeres inscritas por sobre un 43 por ciento de hombres. Acorde con el mismo informe y en cuestiones de tipo de egreso se manejó una cifra de un total de 2,119 mujeres de un total de 3,523 , o sea un 60 por ciento del total general en el nivel de Técnico
Céco do Núcleo de Estudos e Pesquisas sobre Gênero e Direito Centro de Ciências Jurídicas - Universidade Federal da Paraíba V. 6 - No 02 - Ano 2017

171

Superior Universitario, Licenciaturas y

Posgrados en la modalidad escolarizada; y en la modalidad no escolarizada surgió una cifra de 150 mujeres de un total de 256 egresados siendo esto un 59 por ciento del total. Estos altos indicadores con referencia a las mujeres se refuerzan con los representados en materia de titulación, que es la última etapa donde se reflejan todos los esfuerzos realizados durante su proceso académico, y éste manifiesta que en el nivel de Técnico Superior Universitario y Licenciaturas, de un total de 2,374 alumnos, 1,390 son mujeres, o sea, un 59 por ciento del total titulados son mujeres; mientras que en el nivel de Posgrado se maneja un total de 213 titulados, de los cuales 115 son mujeres, representándose este con un 53 por ciento.

\section{CONCLUSIÓN}

La educación es un poder para las mujeres que les permite desarrollar esas aptitudes necesarias para desenvolverse y empoderarse y lograr alcanzar esos espacios de liderazgo, que les permita proveer y establecer herramientas que faciliten el camino a otras y permitan el proceso de inclusión en los sectores 


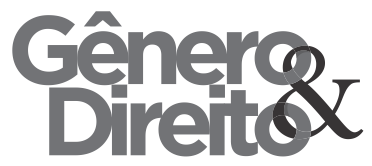

educativos y laborales. Durante el desarrollo de la investigación se observa la lucha incansable de las mujeres y se ven resultados de los notables avances de lo que ellas han logrado y se manifiesta en las expectativas sociales y personales de las mujeres.

Se debe seguir luchando por diseminar la apertura de esos espacios, pues el beneficio redundará para mejorar la sociedad y su desarrollo. Eliminando razgos de discriminación, violencia y menosprecio de las habilidades con que las mujeres cuentan, se les logrará eliminar de los grupos en situación de vulnerabilidad. Estas actitudes y aptitudes retrógradas engendradas por las ideas y tradiciones culturales alientan las preferencias por beneficiar a los hombres en lugar de las mujeres, aunque sus capacidades no estén al mismo nivel.

Razón por la cual, esta lucha debe ser continua pues, a pesar de estos "avances", México todavía presenta bajos niveles de educación, en comparación con los países más desarrollados y es triste ver que los derechos de la mujer en algunas comunidades continúan sin ser respetados de manera igualitaria. Puesto que, aunque el número de mujeres que ingresan al
Céco do Núcleo de Estudos e Pesquisas sobre Gênero e Direito Centro de Ciências Jurídicas - Universidade Federal da Paraíba V. 6 - No 02 - Ano 2017

mercado laboral aumenta año con año, su tasa de participación en el Estado es limitado (según el Instituto Nacional de Estadística y Geografía [INEGI] (2006), un 34.5 por ciento) al igual que en el país (tiene un porcentaje de un 41.4 por ciento), al que se le anexa el trabajo fantasma que es el correspondiente al de las actividades domésticas. La misma desigualdad se manifiesta en el pago de salario, pues las mujeres perciben sólo 51.5 pesos por hora, mientras que un hombre recibe 62.3 pesos (INEGI, (2006).

Está comprobada igual la violencia emocional que viven, la cual impacta enormemente en su desarrollo personal y que por lo general viene acompañada de otros tipos de violencia, pues en el Estado se alcanzó la cifra de un 35.4 por ciento, y la económica de un 25.9 por ciento, junto con la física de un 14 por ciento y sexual en un 6 por ciento y a como se ha comentado principalmente predominan estas situaciones en las comunidades rurales, alejadas de la urbanidad y modernidad (INEGI, (2006).

Estos datos que en cálculos presentados se ven fríos, demuestran la profundidad de la necesidad imperante de continuar con la lucha de la defensa de los 
derechos y libertades de las mujeres, pues el limitar a este factor importante de la sociedad lo único que logra es minimizar el desarrollo de un Estado y afecta enormemente el desenvolvimiento de un país.

\section{BIBLIOGRAFÍA}

Convención Interamericana de Derechos Humanos [CIDH] (1994). Convención Interamericana para prevenir, sancionar y erradicar la Violencia contra la Mujer "Convencion De Belem Do Para". Tratados Multilaterales. Departamento de Derecho Internacional, OEA. Recuperado en http://www.oas.org/juridico/spanish/trata dos/a-61.html

Córdova Osnaya, M., (s. f.). La Mujer Mexicana como Estudiante de Educación Superior. UNAM (México). Psicología para América Latina. Recuperado en https://psicolatina.org/Cuatro/mexicana.ht $\underline{\mathrm{ml}}$

Gayol, Y. (2015). Mujer, igualdad y educación a distancia. U2000: Crónica de la Educación Superior, XXV(893), 1 y 5.
Recuperado

en

https://www.academia.edu/11618990/Muj

$\underline{\text { er_igualdad_y_educacion_a_distancia }}$

Instituto Nacional de Estadística y Geografía [INEGI] (2006). Las mujeres en Tabasco. Estadísticas sobre desigualdad de género y violencia contra las mujeres. Recuperado en http://www.diputados.gob.mx/documento s/Congreso_Nacional_Legislativo/delitos _estados/Las_Mujeres_Tabasco.pdf

Lavalle Urbina, M., (1988). La mujer mexicana a través de los años. Repercusiones históricas y laborales como factores de empuje en la migración internacional. Recuperado en http://catarina.udlap.mx/u_dl_a/tales/docu $\underline{\text { mentos/lri/rojas_g_i/capitulo2.pdf }}$

México Desconocido, (24 de febrero de 2014). Biografía de Sor Juana Inés De la Cruz: 1648 - 1695. Recuperado en https://www.mexicodesconocido.com.mx/ $\underline{\text { sor-juana-ines-de-la-cruz-1648- }}$

\section{1.html}

Morales, T (2015). La mujer y el derecho a la educación. Recuperado: 
Gônerger

http://aion.mx/reportaje/la-mujer-y-el-

derecho-la-educacion

Moreno, M., (2006). Una historia de la educación a distancia en México.

Documento de trabajo para el curso “Teoría y práctica de la Educación a Distancia". Sistema de Universidad Virtual, Universidad de Guadalajara, consultado en línea el 20 de octubre de 2016 ,

en

http://mail.udgvirtual.udg.mx/biblioteca/b itstream/123456789/1355/1/Una_historia _de_la_educacion_a_distancia_en_Mexic $\underline{\text { o.pdf }}$

Nadeskko (2010). Mujeres y educación en México (Nadeskko). Percepciones. Comentarios varios, pensamientos, percepciones, sueños... Recuperado en http://nadeskko.blogspot.mx/2010/04/muj eres-y-educacion-en-mexico-

$\underline{\text { nadeskko.html }}$

Pérez Castro., J., (2014). Avatares y desafíos del Instituto Juárez de Tabasco. Secuencia (2015), 91, enero-abril, 151170. ISSN: 0186-0348. Recuperado en https://www.researchgate.net/publication/
Cico do Núcleo de Estudos e Pesquisas sobre Gênero e Direito Centro de Ciências Jurídicas - Universidade Federal da Paraíba V. 6 - $\mathrm{N}^{\circ} 02$ - Ano 2017

292075761_Avatares_y_desafios_del_Ins

$\underline{\text { tituto_Juarez_de_Tabasco }}$

Pérez Morales, F., Castillo Guzmán, R., Gorrochotegui Salas, M., y Estrada

Sánchez, C. (2008). Rosario María

Gutiérrez Eskildsen: figura histórica de la educación en Tabasco. Semana de

Divulgación y Video Científico.

Universidad Juárez Autónoma de

Tabasco. Recuperado en

http://www.archivos.ujat.mx/dip/divulgac

ion $\% 20 y \% 20$ video\%20cinetifico\%20200

8/DAEA/FPerezM1.pdf

Rodríguez Mancera, M., (s. f.). La

Historia de la Educación de la Mujer en

México. II Congreso Nacional: Estudios

Regionales y la Multidisciplinariedad en

la Historia. Recuperado en

http://filosofia.uatx.mx/memoriasIII/2.pdf

Organización de las Naciones Unidas para la Educación, la Ciencia y la Cultura [UNESCO], (s. f.). Educación para el siglo XIX. Recuperado en http://es.unesco.org/themes/education21st-century

Pedrero-Nieto, G; Badía-Muñoz, G I; (2015). Historia del "Instituto José N. Rovirosa" de Villahermosa, Tabasco, en 
voz de sus protagonistas. Contribuciones

desde Coatepec, () 107-126. Recuperado

de

http://www.redalyc.org/articulo.oa?id=28

\section{$\underline{139198006}$}

Trillo Tinoco, F., (2004). La presencia de las mujeres en la Internet. Tesis licenciatura. Universidad Autónoma de México. Recuperado en file:///C:/Users/josef/Downloads/La_pres encia_de_las_mujeres_en_la_Intern.pdf

Torres Vera, M., Romero Rodríguez, L., (s. f.). Dos proyectos de Educación Superior en el Tabasco Porfiriano. Centro Internacional de Posgrado A. C. XI Congreso Nacional de Investigación Educativa. Historia e Historiografía de la Educación. Recuperado en http://www.comie.org.mx/congreso/mem oriaelectronica/v11/docs/area_09/1653.pd 